\title{
Augmented Renal Clearance in Chinese Intensive Care Unit Patients After Traumatic Brain Injury: Identifying Estimate Glomerular Filtration Rate Cut- off Value as a Risk Factor
}

\section{Zilong Dang}

Department of Pharmacy, The First Hospital of Lanzhou University

Hong Guo

Department of Critical Care Medicine, The First Hospital of Lanzhou University

Bin Li

Department of Critical Care Medicine, The First Hospital of Lanzhou University Maohua Zhen

Department of Neurosurgery, The First Hospital of Lanzhou University, Lanzhou, Gansu, China Jian Liu

Department of Critical Care Medicine, The First Hospital of Lanzhou University

\section{Yuhui Wei}

Department of Pharmacy, The First Hospital of Lanzhou University

\section{Hongyan Qin}

Department of Pharmacy, The First Hospital of Lanzhou University

\section{Zhimin Dou}

Department of Critical Care Medicine, The First Hospital of Lanzhou University

\section{Lei Zhang}

Department of Critical Care Medicine, The First Hospital of Lanzhou University

\section{Lei Zhu}

Department of Critical Care Medicine, The First Hospital of Lanzhou University

\section{Yongqiang Cao}

Department of Critical Care Medicine, The First Hospital of Lanzhou University

\section{Fengjiao Li}

Department of Critical Care Medicine, The First Hospital of Lanzhou University Xinan Wu ( $\nabla$ xinanwu6511@163.com)

Department of Pharmacy, The First Hospital of Lanzhou University, Lanzhou, Gansu, China;

\section{Research}


Keywords: Augmented renal clearance, traumatic brain injury, estimated GFR, risk factors, prevalence, ARCTIC

Posted Date: September 22nd, 2020

DOI: https://doi.org/10.21203/rs.3.rs-78777/v1

License: (c) (1) This work is licensed under a Creative Commons Attribution 4.0 International License. Read Full License 


\section{Abstract}

\section{Background}

Augmented renal clearance (ARC) is common in trauma patients and associated with subtherapeutic antimicrobial concentrations. China has more patients with traumatic brain injury (TBI) than most other countries in the world. However, there were no studies that focus on the ARC in Chinese TBI patients. In this study, we report the incidence of ARC in patients with TBI as well as risk factors for ARC. Besides, we evaluated the ability of four commonly used estimate glomerular filtration rate (eGFR) formulas and other covariates to predict the presence of ARC.

\section{Methods}

The prospective observational study was conducted in a 24-bed tertiary level, university-affiliated hospital, and 54 TBI patients with normal serum creatinine enrolled. We measured 24-h creatinine clearance after admission, and ARC defined as measured creatinine clearance $\geq 130 \mathrm{ml} / \mathrm{min} / 1.73 \mathrm{~m}^{2}$. Demographic variables were then compared, and multivariate analysis was performed. Receiver operating curve, bias, and precision analyses were conducted to examine eGFR and ARCTIC score accuracy in predicting ARC. Multiple logistic regression analysis was performed to estimate the risk factors contributing to ARC.

\section{Results}

The incidence of ARC was $50 \%$. A moderate correlation was shown between measured $\mathrm{Crcl}$ and four eGFR $\left(r_{s} / r\right.$ was $0.590,0.499,0.404$, and 0.518 , respectively). All of the equations significantly underestimated the measured $\mathrm{Crcl}$, especially in the ARC group. Neither had excellent precision for clinical application in this setting. ARCTIC score can't accurately distinguish ARC either. Multivariable analysis displayed that four factors independently correlated to ARC: male, larger BMI, lower serum creatinine, and without a history of hypertension. Further investigation found eGFR higher than the threshold, without a history of hypertension, and/or bigger BMI were independent risk factors for ARC, and they had good accuracy in predicting the occurrence of ARC.

\section{Conclusions}

ARC was frequently observed in Chinese TBI patients. The estimated methods of GFR and ARCTIC score inaccurate and couldn't be directly used as a screening tool to identify ARC in Chinese TBI patients. But if we take the cut-off values of different formulas into account, the estimated GFR could be used as a decisive risk factor in helping to screen the ARC.

\section{Background}

The incidence of ARC(Augmented renal clearance) has been reported to range from 14-85\%[1], depending on the population studied and the cut-off values used for its definition. As to most patients, 
the occurrence of ARC on the first day of ICU admission may predict continued creatinine clearance $(\mathrm{CrCl})$ elevation for one day to several weeks[1,2].

The occurrence of ARC has a very substantial effect on $\beta$-lactam antibiotics PK/PD. Patients with ARC more likely to have very low $\beta$-lactam trough concentrations and the reduced probability of reaching the PK/PD target value at the conventional dosage compared with patients without ARC[2-5]. Treatment failure or worse outcome was more frequent in ARC patients, and ARC was significantly associated with recurrent infections $[6,7]$. Besides, in logically, ARC is likely to promote the emergence of resistance by incompletely treating infection due to lower serum levels.

It has been reported that the incidence of ARC in traumatic brain injury (TBI) patients as high as $85 \%$ [8]. As stated, the rate of nosocomial infections after TBI was 33.3\%[9]. And those cleared by the kidney, like beta-lactam antibiotics, were frequently used as anti-infection therapy drugs. Whether the anti-infection therapy successful is one of the critical factors that influence the prognosis of the patient. China has more patients with TBI than most other countries in the world, making this condition a significant public health concern[10]. Unfortunately, the occurrence of ARC in TBI patients didn't get the deserved attention of the Chinese medical staff. As a result, there have been no studies on ARC in patients after TBI in China, and the incidence and risk factors are unknown, not mention to adjust the dosage of the anti-infection drug after ARC. Therefore, it is of great significance to explore the prevalence and risk factors of ARC in Chinese TBI patients.

Until now, although direct measurement of the glomerular filtration rate (GFR) with exogenous substances such as inulin is the gold standard for the assessment of renal function, it is not routinely performed in intensive care units for practical reasons. Instead, one could measure the $\mathrm{CrCl}$ from a 24 or 8-h urine collection, which can be realized in ICU but would cost much labor. So a replacement method is using the estimated GFR or identifies the risk factors to screen the ARC patient. In Chinese clinical practice, the GFR is most commonly estimated from the serum creatinine ( $\mathrm{SCr}$ ), using various formulas including Cockcroft-Gault, Modified Chinese MDRD Study equation(CMDRD), and the Chronic Kidney Disease Epidemiology Collaboration for Asian people (CKD-EPI-Asian) and Japanese eGRF equation[11].

In the year 2016, Barletta et al. [12] constructed a scoring system, Augmented Renal Clearance in Trauma Intensive Care scoring system(ARCTIC), which was specifically used to predict the ARC in American trauma patients. Whether this scoring system can be applied to Chinese TBI patients is not yet known.

Thus, the primary aim of the study was to explore the incidence of ARC after traumatic brain injury in Chinese patients, meanwhile to assess the accuracy of 4 commonly used formulas and the ARCTIC scoring system in identifying ARC with normal SCr levels. The secondary aim was to determine risk factors for ARC.

\section{Methods}


This prospective, single-center, observational study was conducted in a 24-bed intensive care unit of the First Hospital of Lanzhou University, a tertiary teaching hospital in Gansu Province, China, from January 2019 to November 2019. This study was approved by the institutional ethics committee (LDYYLL2018153), and informed consent was obtained from all participants or a surrogate decision-maker.

\section{Study Population}

Patients with no evidence of renal impairment (admission SCr $>113 \mu \mathrm{mol} / \mathrm{L}$ ), no history of renal replacement therapy, and 18\age $\leq 65$ were enrolled. The exclusion criteria for study admission were as follows: age $<18$ years, pregnancy, suspicion of rhabdomyolysis, and developing AKI during the treatment(2012 KDIGO Clinical Practical Guideline for Acute Kidney Injury).

\section{Data Collection And Definition}

As soon as the patient met the inclusion criteria, 24-hour urine samples and a blood sample were collected for creatinine clearance calculation. Measured $\mathrm{CrCl}$ was then calculated using the standard formula: $\mathrm{CrCl}=(\mathrm{UCr} \times \mathrm{UV}) /(\mathrm{SCr} \times 1440)$, where $\mathrm{UCr}$ (urine creatinine concentration) and $\mathrm{SCr}$ were expressed in $\mu \mathrm{mol} / / \mathrm{I}$ and UV corresponded to the volume of urine in $\mathrm{mL}$. At the same time, the estimated glomerular filtration rate was calculated using different formulas. We calculated eGFR by using following equations:

Cockcroft-Gault equation(CG)[13]:

Males, eGFR(ml/min)=(140-age $) \times$ Weight $/ 72 \times S C r$

Femals, eGFR $(\mathrm{ml} / \mathrm{min})=(140$-age $) \times$ Weight $/ 72 \times \mathrm{SCr} \times 0.85$

Modified Chinese Modification of Diet in Renal Disease Study equation(CMDRD)[14]:

Males, eGFR $(\mathrm{ml} / \mathrm{min})=186 \times \mathrm{SCr}^{-1.154} \times \operatorname{age}^{-0.203} \times 1.233$

Females, eGFR(ml/min $)=186 \times \mathrm{SCr}^{-1.154} \times \mathrm{age}^{-0.203} \times 1.233 \times 0.742$

Chronic Kidney Disease Epidemiology Collaboration equation for Asian people[CKD-EPI-Asian] [15, 16]:

Males, $\mathrm{SCr} \leq 80, \mathrm{eGFR}(\mathrm{mL} / \mathrm{min})=141 \times(\mathrm{SCr} / 0.9)^{-0.411} \times 0.993^{\mathrm{age}} \times 1.057$

Males, SCr囚80, eGFR(mL/min $)=141 \times(\mathrm{SCr} / 0.9)^{-1.209} \times 0.993^{\text {age }} \times 1.057$

Females, $\mathrm{SCr} \leq 62, \mathrm{eGFR}(\mathrm{mL} / \mathrm{min})=144 \times(\mathrm{SCr} / 0.7)^{-0.329} \times 0.993^{\text {age }_{x}} 1.049$

Females, SCr区62, eGFR $(\mathrm{mL} / \mathrm{min})=144 \times(\mathrm{SCr} / 0.7)^{-1.209} \times 0.993^{\mathrm{age} \times 1.049}$

The Japanese eGFR equation(J-eGFR)[17] 
For males: eGFR $(\mathrm{mL} / \mathrm{min})=194 \times \mathrm{SCr}^{-1.094} \times \mathrm{age}^{-0.287}$

For females: eGFR $(\mathrm{mL} / \mathrm{min})=194 \times \mathrm{SCr}^{-1.094} \times$ age $^{-0.287} \times 0.739$

We calculated body surface area by using following equations[18]:

Males, BSA $=0.0057 \times$ Height $+0.0121 \times$ Weight $+0.0882 \rrbracket$

Femals, BSA=0.0073×Height+0.0127×Weight-0.2106》

Patient characteristics, clinical features were extracted from medical records. Medical history of high blood pressure(hereinafter referred to as HBP), Acute Physiology And Chronic Health Evaluation (APACHE) II, Glasgow coma scale(GCS) score on the admission-day were calculated from the charts. Data collection began immediately after obtaining informed consent. It was discontinued for death, development of severe renal impairment (measured $\mathrm{Crcl}<30 \mathrm{~mL} / \mathrm{min}$ ), initiation of renal replacement therapy, and patient consent withdrawal. ARC was defined as a $24-\mathrm{h} \mathrm{Crcl} \geq 130 \mathrm{~mL} / \mathrm{min}$.

\section{Statistical Analysis}

Data that were normally distributed were presented as the means and SDs, and differences between groups were assessed using Student t-tests. Data that were not normally distributed were presented as medians with interquartile ranges, and differences between groups were compared using Mann-Whitney $U$ tests. Categorical variables were presented as numbers with percentages and were analyzed using the $\chi^{2}$ test or Fisher exact tests. Input and a forward conditional logistic regression model was developed to describe risk factors for ARC in multivariate analysis. To evaluate the relationships between eGFR, which was calculated by different equations, and $24 \mathrm{~h}$ creatinine clearance, a receiver operating characteristic curve(ROC) was constructed. The area under the curve and Youden index were also computed to identify the best cut-off value. All statistical analyses were performed using IBM-SPSS (version 26.0) and MedCalc (version 12.7.0.0)statistical software, and differences were considered statistically significant at $\mathrm{P}<0.05$ (2 sided).

The bias and the precision of different formulas compared with the measured $\mathrm{CrCl}$ were evaluated according to Sheiner et[19] by the following equations:

$$
\text { Bias }=\frac{1}{N} \sum_{i=1}^{N}\left(C r l_{\text {estimated }}-C r c l\right)
$$

Precision $=\sqrt{\frac{1}{N} \sum_{i=1}^{N}\left(\mathrm{Crcl}_{\text {estimated }}-\mathrm{Crcl}\right)^{2}}$ 
Residual plots analyzed the agreement between the individual eGFRs by the CG, CMDRD, CKD-EPI-Asian, Japanese eGFR equation, and the measured $\mathrm{CrCl}$ according to the method of Bland and Altman[20]. The odds ratio (OR) and $95 \%$ confidence intervals $(\mathrm{Cl})$ were calculated.

\section{Results}

\section{Baselines Characteristics of Study Subjects}

The characteristics of the enrolled patients are shown in Table 1. We enrolled 54 patients in this study(median age, 50 years [IQR,40.8-56.5], 63.0\% male). Of these, 27 patients(50\%) manifesting ARC. ARC occurred less frequently in patients with $\operatorname{HBP}(3 / 16,5.6 \%)$ in comparison with the overall incidence of $50 \%(27 / 54)$. The ARC group manifests the lower serum creatinine clearance 56 [IQR, 48.0-66.0] comparison with the patient without ARC 65[IQR, 58.0-76.0]. The mean 24-h Crcl of patients with ARC was significantly higher : $175.13 \mathrm{ml} / \mathrm{min} / 1.73 \mathrm{~m}^{2}$ vs $101.35 \mathrm{ml} / \mathrm{min} / 1.73 \mathrm{~m}^{2}$ in patients without ARC (p区 0.001).

\section{Accuracy of eGFR and ARCTIC Score system}

The eGFR of patients with ARC was significantly higher than that of patients without ARC $(p<0.001)$ (Fig. $1 A, B, D)$ except the one calculated by CKD-EPI-Asian formula. Analysis to determine the correlation between ARC and eGFR revealed that a moderate correlation was found between measured Crcl and four calculated eGFR, with a Pearson(r)/Spearman coefficient $\left(r_{s}\right)$ of $0.590,0.499,0.404$ and 0.518 respectively $(\mathrm{p} \otimes 0.01)$ (Fig.2 A-D).

In the ARC subgroup, each formula underestimated $\mathrm{CrCl}$ (Additional eTable 1). For all four methods, a more significant bias and a lower precision were observed in the ARC group (Additional eTable 1).

We also test the ARCTIC score, a predictive model used for screen ARC among the trauma patient. The results showed that a weak positive correlation of $0.269(\mathrm{p} \otimes 0.01)$ between $\mathrm{Crcl}$ and ARCTIC scores. The sensitivity of the scoring system as high as $88.9 \%$, but the specificity only $29.6 \%$ (Additional eTable 2 ).

\section{Tools To Screen ARC}

The following variables were significantly different between patients with and without ARC: serum creatinine( 56.0 vs $65.0, \mathrm{p} \otimes 0.05)$, HBP rate( $3 \%$ vs $13 \%, \mathrm{p} \otimes 0.01)$. Although age, weight, $B M \mathrm{Ml}$, and BSA showed no difference between the two groups, they were closed to substantial(The p-Value below or close to 0.1). Multiple logistic regression analysis was performed for five variables (Age, Gender, BMI, SCr, $\mathrm{HBP}$ ). And the result showed that males, BMI, SCr, and without HBP were independent risk factors for ARC (OR, 8.6, 1.3, 0.9, 0.1; $95 \% \mathrm{Cl}, 1.2-62.8,1.1-1.7,0.9-1.0,0.0-0.4$, respectively) (Additional eTable 3). The results demonstrate male patient does not has HBP, has a lower serum creatinine, and greater BMI may 
have higher risk suffer from ARC. We performed the ROC analysis to evaluate the predictive value of eGFR and ARCTIC score for ARC. The AUROC of eGFR calculated by CG, CMDRD, CKD-EPI-Asian, J-eGFR, and ARCITC score were $0.77(95 \% \mathrm{Cl}, 0.65-0.90), 0.71(95 \% \mathrm{Cl}, 0.57-0.85), 0.63(95 \% \mathrm{Cl}, 0.47-0.78)$, $0.70(95 \% \mathrm{Cl}, 0.56-0.84)$, and $0.59(95 \% \mathrm{Cl}, 0.44-0.75)$ respectively. The optimal cutoff value of each eGFR and the ARCTIC for ARC was 95.69(sensitivity, $88.9 \%$; specificity, $59.3 \%$ ), 123.70 (sensitivity, $81.5 \%$; specificity, $55.6 \%$ ), 94.30(sensitivity, $96.3 \%$; specificity, 40.7\%), 69.31(sensitivity, $96.3 \%$; specificity, 44.4 \%), and 5.5(sensitivity, $88.9 \%$; specificity, $29.6 \%$ ) (Additional eTable 2). The CG, J-eGFR formula had the highest positive $(68.59 \%)$ and negative $(92.31 \%)$ predictive value, respectively. As ROC analysis results showed (Additional eTable 2), only the CG formula presented AUC above 0.75 to detect an ARC, with a cut-off value of $95.69 \mathrm{ml} / \mathrm{min} / 1.73 \mathrm{~m}^{2}$. Comparison of ROC curves of the four estimated formulas show no difference between CG, CMDRD, and Japanese eGFR AUCs (Fig. 4), but revealed a significant difference between AUC of CG and CKD-EPI-Asian $(p<0.05)$.

We further tested logistic regression models with significant covariates, in particular, including the cut-off values of ARC detection by four different equations (Table 2).

When including the cut-off values of four eGFR and other risk factors, like BMI, HBP, to a new model, both more accurate than the formula itself alone to predict the ARC. All the Negelkerke $\mathrm{R}^{2}$ above 0.45 and indicated a good fit of the model (Additional eTable 4). They all had good sensitivity, specificity, PPV, and NPV, especially the specificity, improved dramatically (Additional eTable 4).

\section{Discussion}

To the best of our knowledge, this is the first study that investigated ARC in China adult patients after TBI. Approximately $50 \%$ of patients suffered TBI with normal SCr levels in the early two days of ICU admission manifested ARC. The eGFR in this report, which was calculated by using CG or CMDRD or Japanese eGFR equation, was significantly different between patients with and without ARC. There was a moderate correlation between the measured $\mathrm{Crcl}$ and four calculated GFR, and a weak correlation between $\mathrm{Crcl}$ and ARCTIC score. However, unfortunately, we found that none of the four eGFR formulas or ARCTIC score can accurately predict the occurrence of augmented renal clearance in those with normal serum creatinine. Because all equations tended to underestimate the $\mathrm{Crcl}$ at high creatinine clearance (Additional eTable 1), so directly using eGFR to identify ARC is not advised. Still, eGFR above the optimal cut-off values as a significant risk factor is strongly recommended to helping identify the ARC.

Until recently, ARC has extensively been studied in general and specific ICU populations, like patients with trauma, burns, traumatic brain injury, polytrauma, sepsis, ventilator-associated pneumonia, or hematological malignancy[3]. In the previous studies[8, 21], the researchers regarding $8 \mathrm{~h} \mathrm{Crcl}$ above 130 $\mathrm{ml} / \mathrm{min} / 1.73 \mathrm{~m}^{2}$ as the threshold. In our study, we used a cut-off value for $24 \mathrm{~h} \mathrm{CrCl}$ of $130 \mathrm{~mL} / \mathrm{min}$ or greater per $1.73 \mathrm{~m}^{2}$ to define ARC. Our results corroborate the data in other literature, reporting ARC prevalence between $14 \%$ and $85 \%[1]$. 
Historically, attention was mostly paid to the decline of renal function and the corresponding necessity of dose adjustments for drugs cleared by the kidney. Recently, the clinical importance of ARC is gradually realized by medical workers, which can be confirmed by the fact that some studies found ARC could affect the pharmacokinetics of many frequently prescribed medications, especially those mainly renally cleared antibacterials. And a number of studies have shown that ARC is a risk factor for not attaining targeted PK/PD targets of antibiotics, thereby causing a potential consequence of therapeutic failure $[2,4$, 5] and recurrent infections of the patients[7]. Specifically, these findings remind the clinician that a 'one size fits all' approach to drug dosing in critical illness is flawed and requires adjustment for some variables, least of which is the diagnostic category. Nevertheless, antimicrobial efficacy is not readily clinically evaluable, making underdosing substantially less visible. Thus, an appropriate assessment of renal function is compulsory for identifying patients displaying $A R C[7,11]$.

Unfortunately, as measuring $24 \mathrm{~h}$ urinary $\mathrm{CrCl}$ is labor-intensive, as it is not routinely performed in ICU patients. Hence, we tested the identification ability of the ARC of 4 popularly used mathematical equations by the Chinese physician, but none of them can show acceptable sensitivity and specificity. Consider to pathophysiological changes of the traumatic brain injury patients and eGFR formulas that were primarily designed for those non-critically ill patients; the result was not a surprise. In the previous study, ARCTIC was constructed by Jeffrey F. Barletta[12] as a useful tool to help identify the ARC in trauma people, which showed an excellent predictive ability. However, our results demonstrated that the ARCTIC score was not accurate when used to identify ARC in Chinese TBI patients. This finding is most likely due to the different injury mechanisms and ethnicities of patients included in the study.

In the absence of measured $\mathrm{CrCl}$ in critically ill patients in daily routine practice and as frequently used eGFR formulas fail to assess renal function, especially for increased renal clearance, evaluating actual kidney function is difficult in this population[22]. Therefore, the identification of predictors for ARC can help the clinicians in recognizing ARC.

Characteristics of ARC patients, like younger age, male sex, bigger BSA, polytrauma, et al., have been extensively described in ICU patients[1, 23]. In this study, we found that male sex, higher BMI, lower serum creatinine, and without a history of HBP were independent risk factors for ARC. When taking the cut-off values of the different formulas into account, the eGFR above the threshold, without HBP, and/or combine with bigger BMI, could be used to identify the ARC more precisely than eGRF alone. The patients with a history of HBP didn't show ARC could be explained by the fact that the HBP does harm to the kidney, and which reduced the GFR and renal tubule excretion. But due to the limited HBP patient number in our study, the results should be interpreted carefully. As to gender, our research found that male patients had a very high risk(OR:8.6[1.2-62.8]) suffer ARC, which was consistent with the found of Claus et al. [6]. But there were also contrary findings, Udy et al. [24]and Minville et al. [25] demonstrated that gender was not a significant covariate predicting ARC. We also found that a bigger BMI was an independent risk factor for ARC, which was exactly contrary to the result of Grootaert et al.[26]. The possible reason for this result is that a considerable portion of the patients included in our study is construction workers. Therefore, high BMI doesn't mean that these patients are obese, but high muscle content and good kidney preserve. 
In contrast, the age of patients has been confirmed by other researchers closely related to the occurrence of ARC in critically ill[ $[23,27]$. Still, it did not differ significantly between our ARC versus the non-ARC group. The main reason maybe was the patients in our study were older.

This study has some limitations. First, this was a single-center study with a limited number of cases. Second, the gold standard for the assessment of renal function is by use of an exogenous marker such as inulin or radioisotopes, which undergoing only glomerular filtration without tubular secretion, was not performed in this study, so it is impossible to assess the actual GFR. Therefore, further studies are needed to address the limitations of this study.

\section{Conclusions}

ARC is frequently observed in Chinese traumatic brain injury patients. The estimated methods of eGFR inaccurate and couldn't be directly used as a screening tool to identify high $\mathrm{CrCl}$, as is the ARCTIC score. Still, if we treat the cut-off values of eGFR as a risk factor and combine with other characteristics, like lager BMI, and without HBP, it could be used as a helpful tool to screen the patients with ARC.

\section{Abbreviations}

IQR: Interquartile range; SD: standard deviation; OR: Odds ratio; 95\% Cl: Confidence interval; ARC: Augmented renal clearance; TBI: traumatic brain injury ; eGFR: estimate glomerular filtration rate; ARCTIC: Augmented Renal Clearance in Trauma Intensive Care; Crcl: creatinine clearance; SCr: serum creatinine; HBP: Medical history of high blood pressure; CMDRD: Modified Chinese MDRD Study equation; CKD-EPIAsian: Chronic Kidney Disease Epidemiology Collaboration for Asian people; J-eGFR: Japanese stimate glomerular filtration rate; AKI: Acute Kidney Injury; CG: Cockcroft-Gault; APACHE II: Acute Physiology And Chronic Health Evaluation II; GCS: Glasgow coma scale; ROC: receiver operating characteristic curve; PPV positive predictive value, NPV negative predictive value;

\section{Declarations}

\section{Acknowledgments}

The authors would like to acknowledge the nurse team (Department of Critical Care Medicine The First Hospital of Lanzhou University, Lanzhou, Gansu, China) for their contribution to collect the urine and serum for $24 \mathrm{~h}$ Crcl determination.

\section{Authors' contributions}

ZLD, HG, and XAW contribute to the literature search, study design, data collection, data analysis, data interpretation, and writing. $\mathrm{BL}, \mathrm{MHZ}, \mathrm{JL}$, and $\mathrm{YHWcontributed} \mathrm{to} \mathrm{the} \mathrm{literature} \mathrm{search,} \mathrm{study} \mathrm{design,} \mathrm{data}$ analysis, data interpretation, critical revision, and rewriting of the manuscript. HYQ, ZMD, LZ, LZ, YQC, and 
FJL contributed to the critical review and rewriting of the paper. XAW directed the study team. All authors approved the final article.

\section{Funding}

Gansu Provincial Department of Education Higher School Innovation Ability Improvement Project (2019B002); First Hospital of Lanzhou University Hospital Fund (Idyyyn2018-04); Chengguan District Science and Technology Plan Project(2020SHFZ0033); WU JIE PING medical foundation (320.6750.2020-04-42).

\section{Availability of data and materials}

Please contact the authors for data requests.

\section{Ethics approval and consent to participate}

The institutional ethics committee of The First Hospital of Lanzhou University approved this study (LDYYLL2018-153), and informed consent was obtained from all participants or a surrogate decisionmaker.

\section{Consent for publication}

N/A

\section{Competing interests}

The authors declare that they have no competing interests.

\section{Author details}

${ }^{1}$ Department of Pharmacy, The First Hospital of Lanzhou University, Lanzhou, Gansu, China;

${ }^{2}$ Department of Critical Care Medicine, The First Hospital of Lanzhou University, Lanzhou, Gansu, China;

${ }^{3}$ Department of Neurosurgery, The First Hospital of Lanzhou University, Lanzhou, Gansu, China;

\section{References}

1. Mahmoud SH, Shen C: Augmented Renal Clearance in Critical Illness: An Important Consideration in Drug Dosing. Pharmaceutics 2017, 9(3):36. 
2. Huttner A, Von Dach E, Renzoni A, Huttner BD, Affaticati M, Pagani L, Daali Y, Pugin J, Karmime A, Fathi $\mathrm{M}$ et al: Augmented renal clearance, low beta-lactam concentrations and clinical outcomes in the critically ill: an observational prospective cohort study. Int J Antimicrob Agents 2015, 45(4):385392.

3. Sime FB, Udy AA, Roberts JA: Augmented renal clearance in critically ill patients: etiology, definition and implications for beta-lactam dose optimization. Curr Opin Pharmacol 2015, 24:1-6.

4. Carlier M, Carrette S, Roberts JA, Stove V, Verstraete A, Hoste E, Depuydt P, Decruyenaere J, Lipman J, Wallis SC et al: Meropenem and piperacillin/tazobactam prescribing in critically ill patients: does augmented renal clearance affect pharmacokinetic/pharmacodynamic target attainment when extended infusions are used? Crit Care 2013, 17(3):R84.

5. Udy AA, Varghese JM, Altukroni M, Briscoe S, McWhinney BC, Ungerer JP, Lipman J, Roberts JA: Subtherapeutic initial beta-lactam concentrations in select critically ill patients: association between augmented renal clearance and low trough drug concentrations. Chest 2012, 142(1):30-39.

6. Claus BO, Hoste EA, Colpaert K, Robays H, Decruyenaere J, De Waele JJ: Augmented renal clearance is a common finding with worse clinical outcome in critically ill patients receiving antimicrobial therapy. J Crit Care 2013, 28(5):695-700.

7. Carrie C, Bentejac M, Cottenceau V, Masson F, Petit L, Cochard JF, Sztark F: Association between augmented renal clearance and clinical failure of antibiotic treatment in brain-injured patients with ventilator-acquired pneumonia: A preliminary study. Anaesth Crit Care Pain Med 2018, 37(1):35-41.

8. Udy A, Boots R, Senthuran S, Stuart J, Deans R, Lassig-Smith M, Lipman J: Augmented creatinine clearance in traumatic brain injury. Anesth Analg 2010, 111(6):1505-1510.

9. Kourbeti IS, Vakis AF, Papadakis JA, Karabetsos DA, Bertsias G, Filippou M, loannou A, Neophytou C, Anastasaki M, Samonis G: Infections in traumatic brain injury patients. Clin Microbiol Infect 2012, 18(4):359-364.

10. Jiang J-Y, Gao G-Y, Feng J-F, Mao Q, Chen L-G, Yang X-F, Liu J-F, Wang Y-H, Qiu B-H, Huang X-J: Traumatic brain injury in China. The Lancet Neurology 2019, 18(3):286-295.

11. Ruiz S, Minville V, Asehnoune K, Virtos M, Georges B, Fourcade O, Conil JM: Screening of patients with augmented renal clearance in ICU: taking into account the CKD-EPI equation, the age, and the cause of admission. Ann Intensive Care 2015, 5(1):49.

12. Barletta JF, Mangram AJ, Byrne M, Sucher JF, Hollingworth AK, Ali-Osman FR, Shirah GR, Haley M, Dzandu JK: Identifying augmented renal clearance in trauma patients: Validation of the Augmented Renal Clearance in Trauma Intensive Care scoring system. J Trauma Acute Care Surg 2017, 82(4):665-671.

13. Cockcroft DW, Gault MH: Prediction of creatinine clearance from serum creatinine. Nephron 1976, 16(1):31-41.

14. Collaboration Cel: Modification and evaluation of MDRD estimating equation for Chinese patients with chronic kidney disease. Chin J Nephrol 2006, 22(10):589-595. 
15. Levey AS, Stevens LA, Schmid CH, Zhang YL, Castro AF, 3rd, Feldman HI, Kusek JW, Eggers P, Van Lente F, Greene T et al: A new equation to estimate glomerular filtration rate. Ann Intern Med 2009, 150(9):604-612.

16. Stevens LA, Claybon MA, Schmid CH, Chen J, Horio M, Imai E, Nelson RG, Van Deventer M, Wang HY, Zuo L et al: Evaluation of the Chronic Kidney Disease Epidemiology Collaboration equation for estimating the glomerular filtration rate in multiple ethnicities. Kidney Int 2011, 79(5):555-562.

17. Kawano Y, Morimoto S, Izutani Y, Muranishi K, Kaneyama H, Hoshino K, Nishida T, Ishikura H: Augmented renal clearance in Japanese intensive care unit patients: a prospective study. $J$ Intensive Care 2016, 4:62.

18. Stevenson PH: Height. weight. surface formula for the estimation of surface area in Chinese subjects. Chinese J Physiol 1937, 12:327-330.

19. Sheiner LB, Beal SL: Some suggestions for measuring predictive performance. Journal of pharmacokinetics and biopharmaceutics 1981, 9(4):503-512.

20. Bland JM, Altman DG: Statistical methods for assessing agreement between two methods of clinical measurement. Lancet 1986, 1(8476):307-310.

21. Udy AA, Jarrett P, Lassig-Smith M, Stuart J, Starr T, Dunlop R, Deans R, Roberts JA, Senthuran S, Boots $\mathrm{R}$ et al: Augmented Renal Clearance in Traumatic Brain Injury: A Single-Center Observational Study of Atrial Natriuretic Peptide, Cardiac Output, and Creatinine Clearance. J Neurotrauma 2017, 34(1):137-144.

22. Grootaert V, Willems L, Debaveye Y, Meyfroidt G, Spriet l: Augmented renal clearance in the critically ill: how to assess kidney function. Ann Pharmacother 2012, 46(7-8):952-959.

23. Udy AA, Roberts JA, Shorr AF, Boots RJ, Lipman J: Augmented renal clearance in septic and traumatized patients with normal plasma creatinine concentrations: identifying at-risk patients. Crit Care 2013, 17(1):R35.

24. Udy AA, Baptista JP, Lim NL, Joynt GM, Jarrett P, Wockner L, Boots RJ, Lipman J: Augmented renal clearance in the ICU: results of a multicenter observational study of renal function in critically ill patients with normal plasma creatinine concentrations. Crit Care Med 2014, 42(3):520-527.

25. Minville V, Asehnoune K, Ruiz S, Breden A, Georges B, Seguin T, Tack I, Jaafar A, Saivin S, Fourcade 0 et al: Increased creatinine clearance in polytrauma patients with normal serum creatinine: a retrospective observational study. Crit Care 2011, 15(1):R49.

26. Grootaert V, Spriet I, Decoutere L, Debaveye Y, Meyfroidt G, Willems L: Augmented renal clearance in the critically ill: Fiction or fact? Int J Clin Pharm 2012, 34:143.

27. Akers KS, Niece KL, Chung KK, Cannon JW, Cota JM, Murray CK: Modified Augmented Renal Clearance score predicts rapid piperacillin and tazobactam clearance in critically ill surgery and trauma patients. J Trauma Acute Care Surg 2014, 77(3 Suppl 2):S163-170.

\section{Tables}


Table 1 Demographic and laboratory data

\begin{tabular}{|c|c|c|c|c|}
\hline Variable & $\begin{array}{l}\text { All } \\
\text { Patients }(n=54)\end{array}$ & $\begin{array}{l}\text { Patients with } \\
\text { ARC }(n=27)\end{array}$ & $\begin{array}{l}\text { Patients without } \\
\text { ARC }(n=27)\end{array}$ & $\begin{array}{l}\mathrm{p} \\
\text { Value }\end{array}$ \\
\hline Age, median(IQR) & $\begin{array}{l}50.0(40.8- \\
56.5)\end{array}$ & $49.0(33.0-54.0)$ & $51.0(43.0-60.0)$ & 0.174 \\
\hline Male, n(\%) & $34(63.0)$ & 18(33.3) & $16(29.6)$ & 0.573 \\
\hline Height(m), mean(SD) & $1.69(0.08)$ & $1.7(0.08)$ & $1.68(0.08)$ & 0.613 \\
\hline Weight(Kg), median(IQR) & $\begin{array}{l}65.0(60.0- \\
75.0)\end{array}$ & $70.0(60.0-76.0)$ & $62.0(60.0-70.0)$ & 0.051 \\
\hline $\begin{array}{l}\text { Body mass index }\left(\mathrm{kg} / \mathrm{m}^{2}\right) \text {, } \\
\text { mean(SD) }\end{array}$ & $23.83(3.85)$ & $24.68(3.89)$ & $22.97(3.68)$ & 0.103 \\
\hline $\begin{array}{l}\text { Body surface area }\left(\mathrm{m}^{2}\right) \text {, } \\
\text { median(IQR) }\end{array}$ & $\begin{array}{l}1.83(1.76- \\
1.98)\end{array}$ & $1.9(1.8-2.0)$ & $1.8(1.7-1.9)$ & 0.073 \\
\hline $\begin{array}{l}\text { Serum albumin }(\mathrm{g} / \mathrm{L}) \text {, } \\
\text { mean(SD) }\end{array}$ & $38.6(6)$ & $38.4(6.4)$ & $38.75(5.63)$ & 0.816 \\
\hline Hemoglobin(g/L), mean (SD) & $130.4(23.4)$ & $130(20.4)$ & $130.7(26.4)$ & 0.913 \\
\hline $\begin{array}{l}\text { Serum Creatinine }(\mu \mathrm{mol} / \mathrm{L}) \text {, } \\
\text { median(IQR) }\end{array}$ & $\begin{array}{l}61.5(52.8- \\
72.8)\end{array}$ & $56.0(48.0-66.0)$ & $65.0(58.0-76.0)$ & $\mathbb{\nabla 0 . 0 5}$ \\
\hline Fluid balance(ml), mean(SD) & $557(1166)$ & $638(1130)$ & $476(1216)$ & 0.613 \\
\hline $\begin{array}{l}\text { Mean urine output }(\mathrm{ml} / \mathrm{kg} / \mathrm{h}) \text {, } \\
\text { median(IQR) }\end{array}$ & $1.4(1.0-2.0)$ & $1.4(1.1-1.9)$ & $1.4(1.0-2.2)$ & 0.710 \\
\hline $\begin{array}{l}24 \mathrm{~h} \mathrm{Crcl}\left(\mathrm{ml} / \mathrm{min} / 1.73 \mathrm{~m}^{2}\right) \text {, } \\
\text { mean }(\mathrm{SD})\end{array}$ & $138.24(44.07)$ & $175.13(28.73)$ & $101.35(17.56)$ & 0.001 \\
\hline $\begin{array}{l}\mathrm{PMH}-\text { High blood pressure, } \\
\mathrm{n}(\%)\end{array}$ & $16(29.6)$ & $3(5.6)$ & $13(24.1)$ & $\mathbb{\nabla 0 . 0 1}$ \\
\hline APACHE II, mean(SD) & $14(4)$ & $14(4)$ & $14(5)$ & 0.900 \\
\hline $\begin{array}{l}\text { First record GCS score, } \\
\text { median(IQR) }\end{array}$ & $8(6-11)$ & $8(6-12)$ & $8(6-11)$ & 0.910 \\
\hline Extradural hematoma, n(\%) & $12(22.2)$ & $4(7.4)$ & $8(14.8)$ & 0.327 \\
\hline Subdural hematoma, $\mathrm{n}(\%)$ & $29(53.7)$ & $15(27.8)$ & $14(25.9)$ & 0.785 \\
\hline $\begin{array}{l}\text { Traumatic subarachnoid } \\
\text { hemorrhage, } n(\%)\end{array}$ & $51(94.4)$ & $27(50.0)$ & $24(44.4)$ & 0.075 \\
\hline Base of skull fracture, $\mathrm{n}(\%)$ & $11(20.4)$ & $6(11.1)$ & $5(9.3)$ & 0.735 \\
\hline Extracranial trauma, $\mathrm{n}(\%)$ & $13(24.1)$ & $6(11.1)$ & $7(13.0)$ & 0.750 \\
\hline
\end{tabular}


PMH, past medical history.

Table 2 Logistic regression for measured creatinine clearance greater than $130 \mathrm{ml} / \mathrm{min} / 1.73 \mathrm{~m}^{2}$

\begin{tabular}{|c|c|c|c|}
\hline ARC & B & $\mathrm{p}$ Value & Odd ratio(IC $95 \%)$ \\
\hline \multicolumn{4}{|l|}{ (A) Taking into account CG equation } \\
\hline High blood pressure & -2.02 & 0.012 & $0.13(0.03-0.65)$ \\
\hline ARC detection by $C G\left(\varangle 95.69 \mathrm{ml} / \mathrm{min} / 1.73 \mathrm{~m}^{2} \square\right.$ & 2.46 & 0.002 & $11.72(2.56-53.63)$ \\
\hline \multicolumn{4}{|l|}{ (B) Taking into account CMDRD equation } \\
\hline $\mathrm{BMI}$ & 0.34 & 0.005 & $1.40(1.10-1.78)$ \\
\hline High blood pressure & -2.92 & 0.002 & $0.05(0.01-0.35)$ \\
\hline 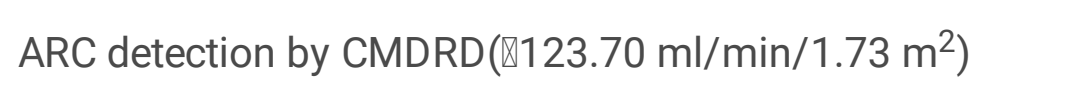 & 2.51 & 0.004 & $12.27(2.20-68.63)$ \\
\hline \multicolumn{4}{|l|}{ (C) Taking into account CKD-EPI-Asian equation } \\
\hline $\mathrm{BMI}$ & 0.28 & 0.010 & $1.32(1.07-1.64)$ \\
\hline High blood pressure & -2.38 & 0.010 & $0.09(0.02-0.56)$ \\
\hline ARC detection by CKD-EPI-Asian $\left(\varangle 94.30 \mathrm{ml} / \mathrm{min} / 1.73 \mathrm{~m}^{2} \square\right.$ & 3.06 & 0.010 & $21.43(2.08-220.37)$ \\
\hline \multicolumn{4}{|l|}{ (D) Taking into account Japanese equation } \\
\hline $\mathrm{BMI}$ & 0.32 & 0.007 & $1.37(1.09-1.73)$ \\
\hline High blood pressure & -2.08 & 0.020 & $0.13(0.02-0.72)$ \\
\hline 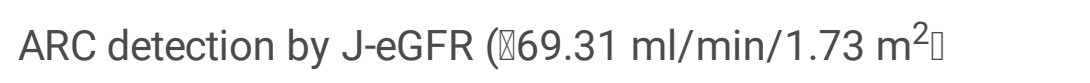 & 3.45 & 0.008 & $31.45(2.51-393.96)$ \\
\hline
\end{tabular}

\section{Figures}


A

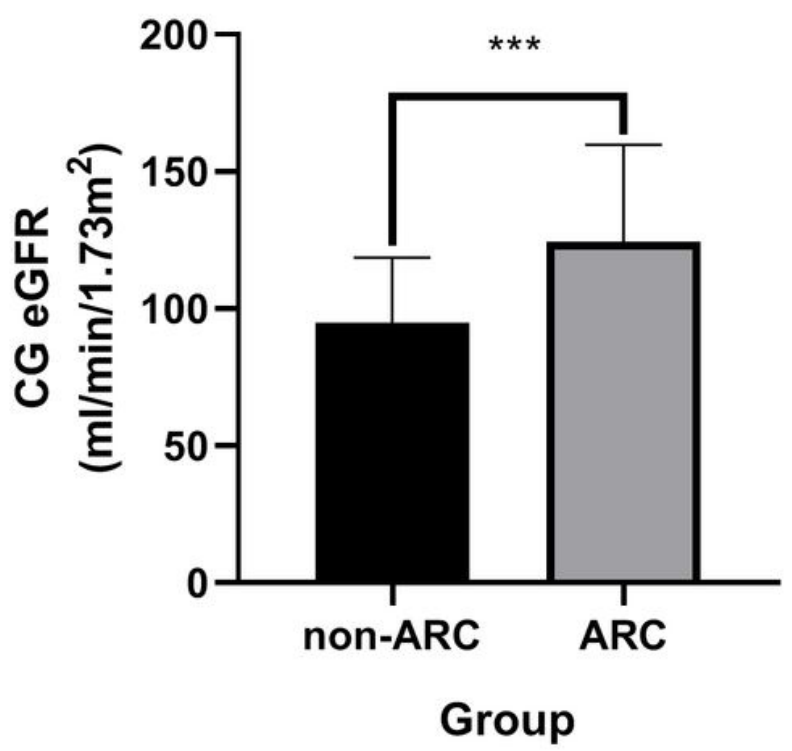

C

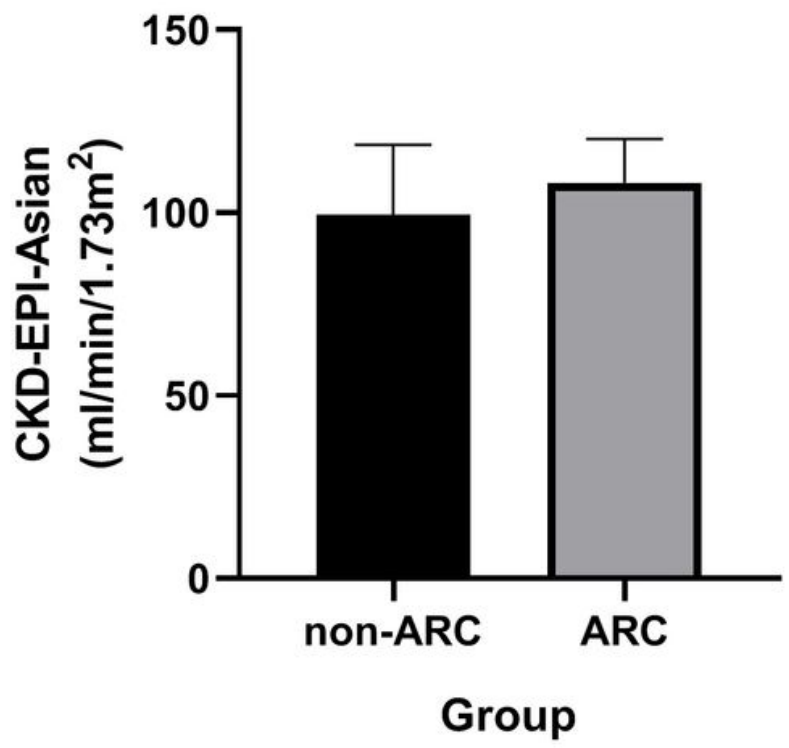

B

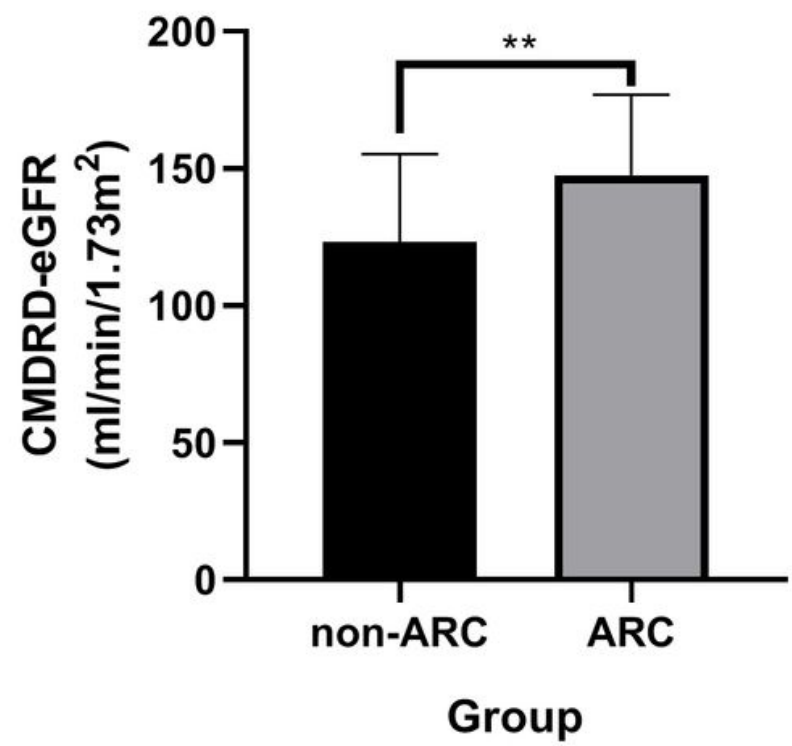

D

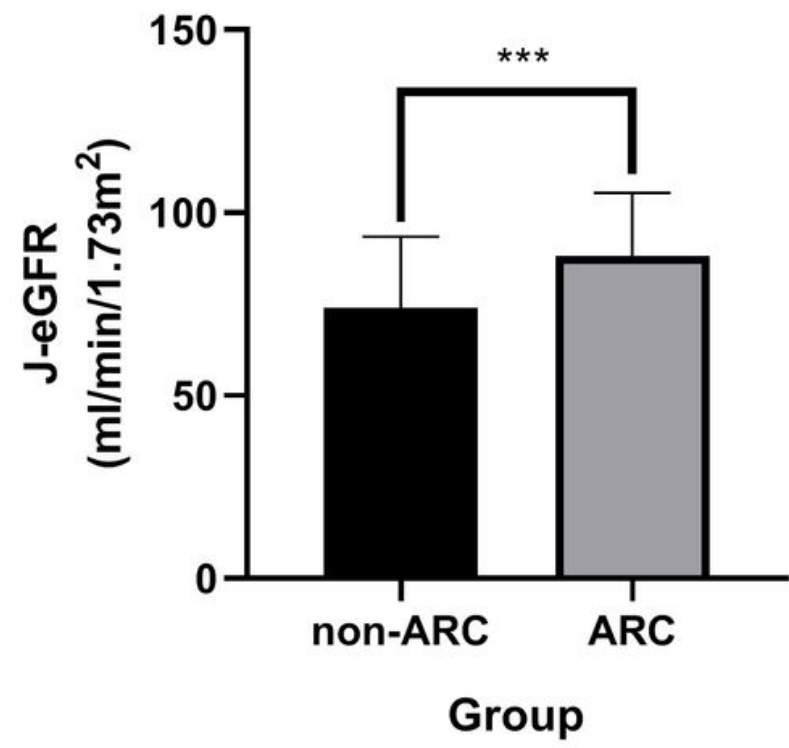

Figure 1

Comparison of the eGFR in patients with and without augmented renal clearance (ARC). The eGFR calculated by CG and CMDRD and Japanese eGFR formula in patients with ARC was significantly higher than that in patients without ARC $(* \star * p<0.001, * * p<0.01)$. 
A

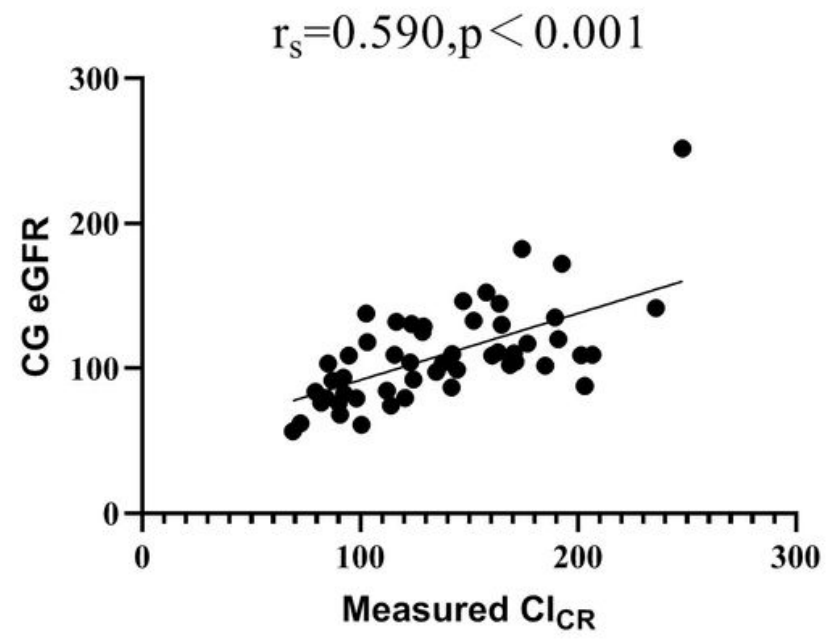

C

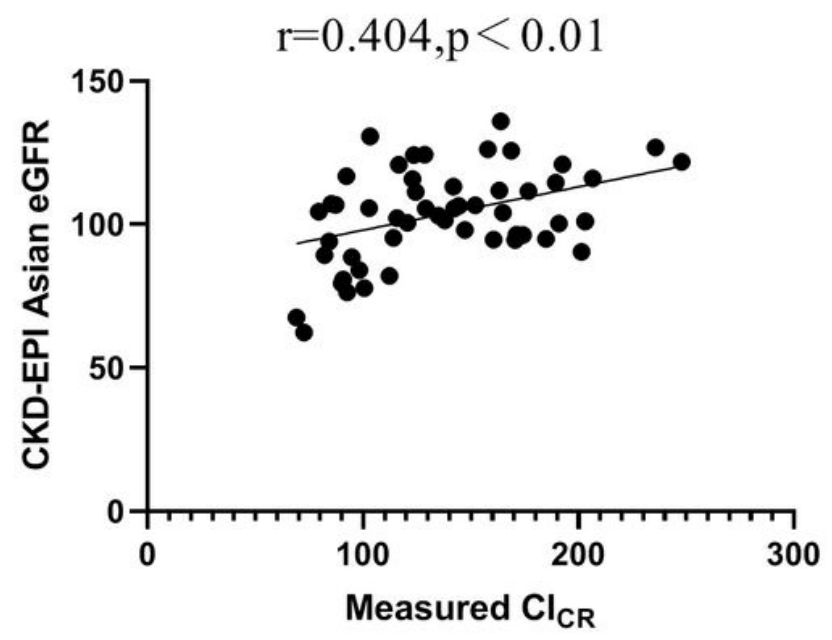

B

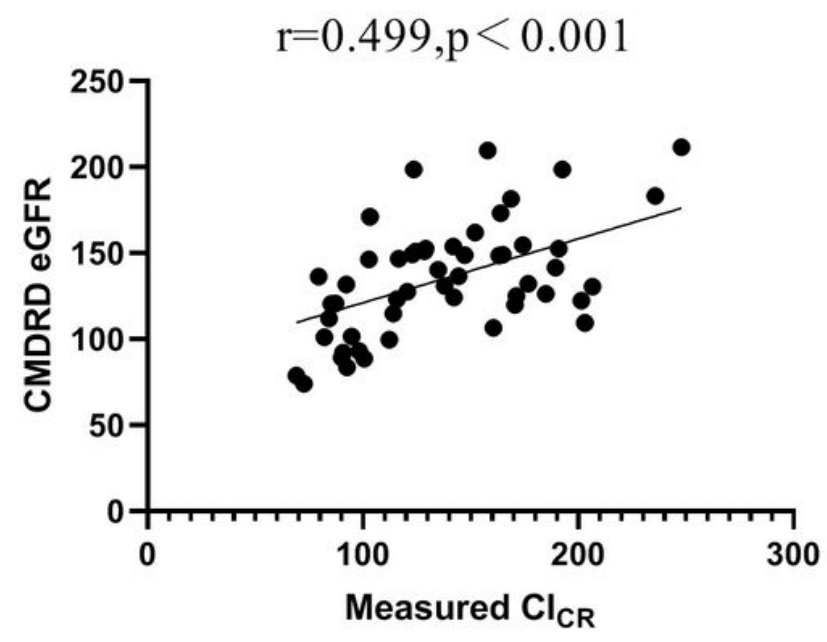

D

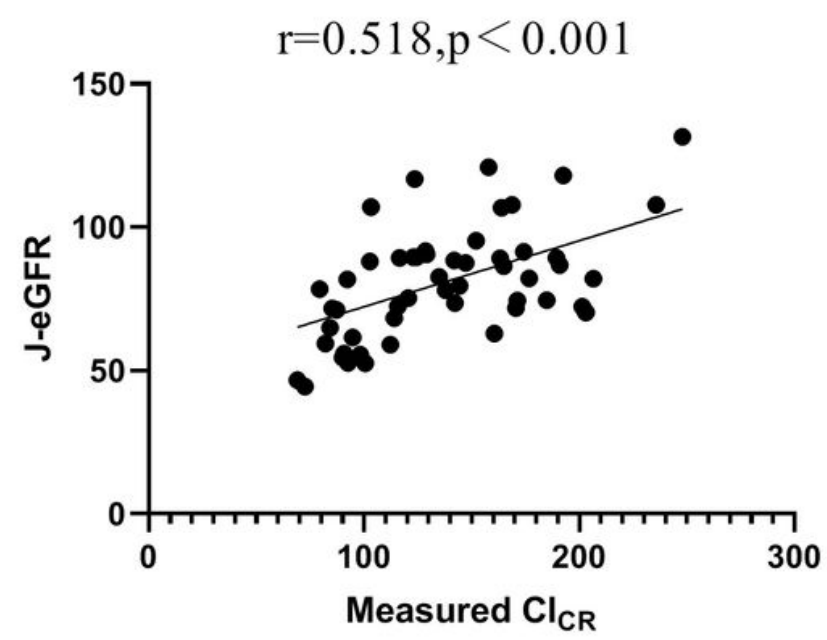

Figure 2

Correlation between the measured $24 \mathrm{~h}$ creatinine clearance $(\mathrm{Crcl})$ and eGFR. A moderate correlation was found between measured $\mathrm{Crcl}$ and eGFR. 

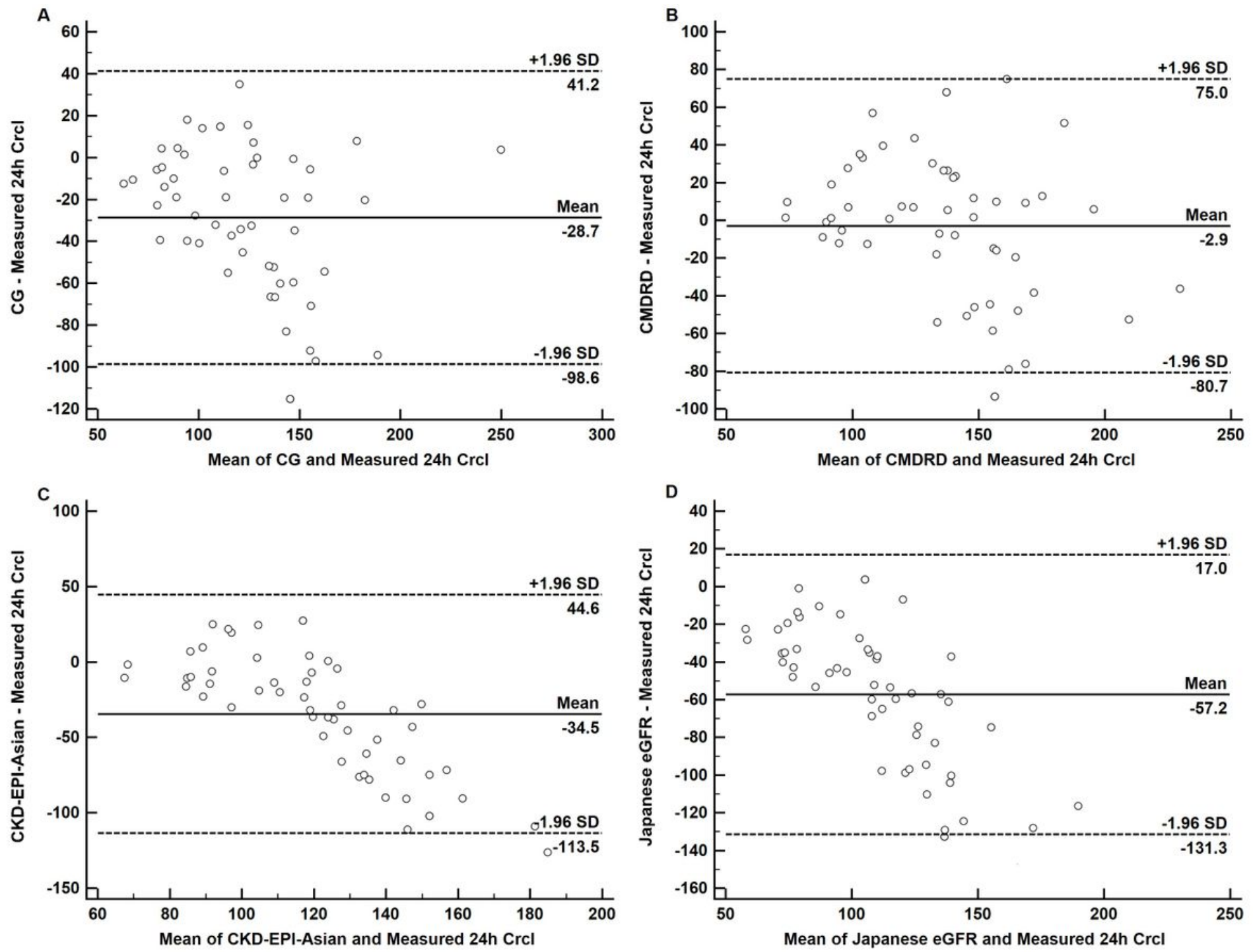

\section{Figure 3}

Measures of agreement between the measured $24 \mathrm{~h}$ Crcl and eGFR. The solid line indicated the mean of the difference between the results of the eGFR and measured $24 \mathrm{~h} \mathrm{Crcl}$. The dashed line shows the $95 \%$ limits of agreement and almost all of eGFR located in the $95 \%$ limits of agreement. 


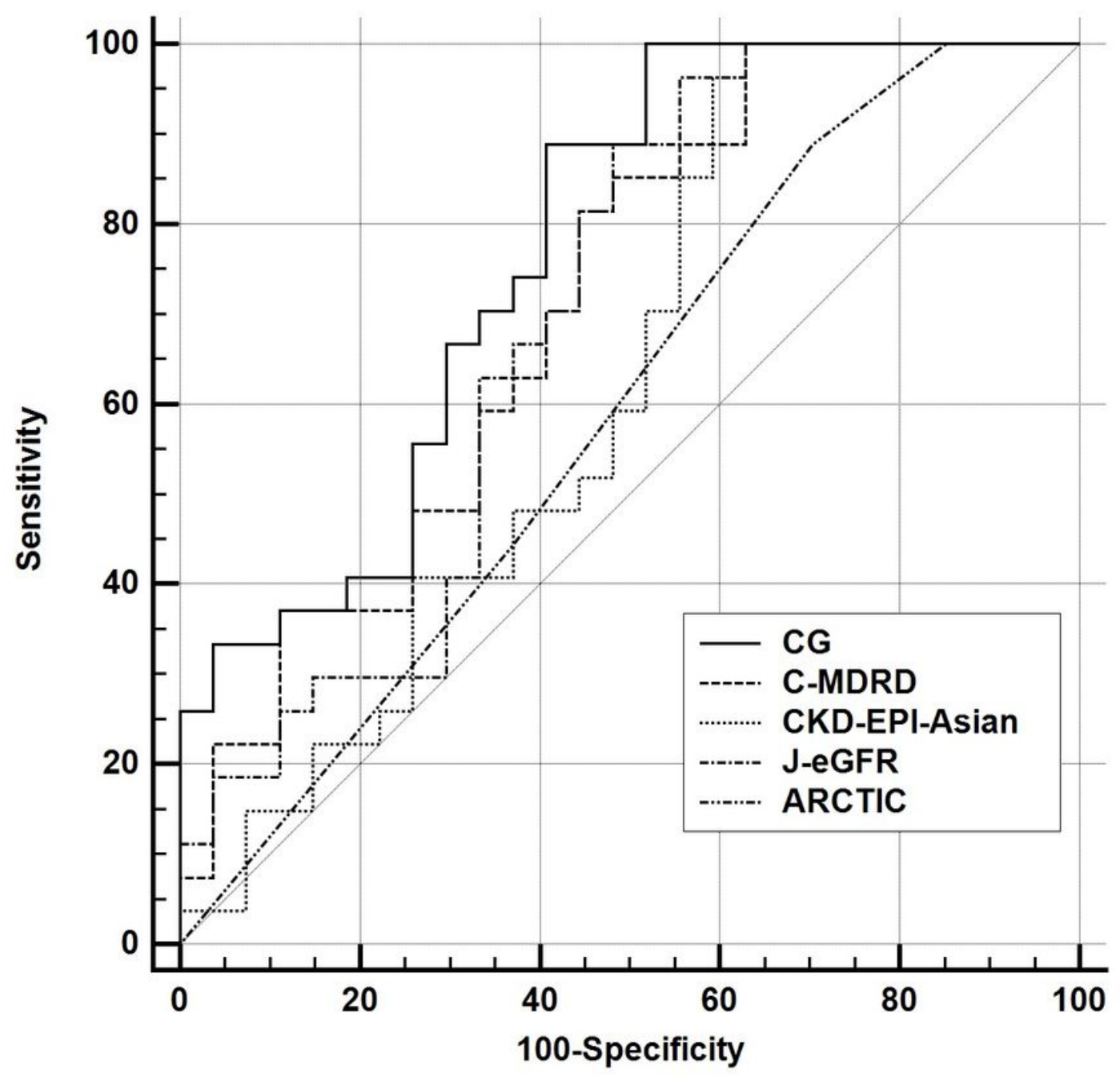

Figure 4

Comparison of ROC curves of four estimated GFR formulas and ARCTIC to detect ARC.

\section{Supplementary Files}

This is a list of supplementary files associated with this preprint. Click to download.

- Additionalfile4.doc

- Additionalfile3.doc

- Additionalfile2.doc

- Additionalfile1.doc 